\title{
SHARING HIGH-RESOLUTION MODELS AND INFORMATION ON WEB: THE WEB MODULE OF BIM3DSG SYSTEM
}

\author{
F. Rechichi ${ }^{\text {a, }}$, A. Mandelli ${ }^{\text {a }}$, C. Achille ${ }^{\text {a }}$, F. Fassi $^{\text {a }}$ \\ ${ }^{\text {a }}$ Politecnico di Milano, A.B.C - Dep. of Architecture, Built environment and Construction engineering, Milano, Italy - \\ (fabrizio.rechichi, alessandro.mandelli, cristiana.achille, francesco.fassi)@polimi.it
}

\author{
Commission V, WG V/4
}

KEY WORDS: BIM, WEB-BIM, BIM3DSG, 3D models, reality based modelling, sharing, big data, WebGL

\begin{abstract}
:
BIM3DSG system is described here. It is an ad hoc designed BIM system created for Cultural Heritage applications. It proposes some solutions to solve some issues related to the use of BIM in this field. First, it tries to resolve the problem of managing huge, complex, high resolution and heterogeneous 3D models, and then it offers a practical, easy and efficient solution for a wide sharing of data and information.
\end{abstract}

\section{INTRODUCTION}

Nowadays, it is the era of explosion of 3D technologies in the field of Building Heritage: for example, laser scanning and photogrammetry are today commonly used in professional activities. The modeling software begin to be more user friendly for the public; they offer very easy and performing tools to elaborate large sets of 3D survey data (dense point clouds and meshes).

Moreover, operators very well understand the potentiality of BIM systems as support for maintenance of buildings. These systems are not only well known and widely spread in the world of construction, but they are becoming necessary in the complicate world of $\mathrm{CH}$ (Cultural Heritage), even if not yet widely used.

There are two main issues to be handled to ensure a future employ of these systems in $\mathrm{CH}$ field: the need to i) manage huge and complex reality based models and many additional information and ii) share 3D data widely and in an easy way (Fassi, at al., 2015).

The adopted solution is to create a BIM system able to manage complex models and dynamic big data, with the ability to interconnect a modeling software with the web.

\section{METHODS AND SYSTEMS FOR THE MANAGEMENT AND THE VISUALIZATION OF COMPLEX THREE-DIMENSIONAL MODELS}

\subsection{State of Art}

We can distinguish three different categories of software related to 3D models; for now, each of them is able to solve only one of the two issues described above, but not both together.

2.1.1 3D modeling software: many software (as for example 3DStudio, AutoCad, Rhinoceros, etc) can manage reality based 3D products. They can handle objects characterized by high complexity, great accuracy, high-resolution and heterogeneous features (both line based and surface based at the same time). Normally, they are directly created from 3D dense point clouds, coming from survey. They have some additional features for extracting metric information, creating mesh surfaces and applying accurate re-topology in order to create final usable models, but they lack in tools for comparing the resulting elements with the original acquired data, in order to estimate the accuracy of the modelling phase.

2.1.2 BIM software: BIM software provide, on paper, a further step: the possibility to manage 3D buildings models as the base for an hard structured information system, namely connecting digital models with different kind of information useful for building management, with the possibility to perform queries and simulations on them. Many researches are focalizing their attention in order to define the ideal data structure for all $\mathrm{CH}$ cases of study; however, it is still very difficult to find it because each situation is very different from others, having particular and unique needs and history. For this reason, hard structured information system cannot be actually used in $\mathrm{CH}$ field, that requires more dynamic systems, able to self adapt to every case, covering all possible needs.

Revit, for example, is one of best known "building design software specifically built for Building Information Modeling (BIM), with features for architectural design, MEP and structural engineering, and construction" based on "parametric components" (Revit, 2015). The use of parametric models is the main reason why they are not very suitable to manage reality based 3D: they cannot be used to view and describe the proper shape (single details and high representation scale) of many different objects at the same time.

aSPECT 3D is instead a photogrammetric software that can be linked with an external PostgreSQL database, which can be used to "systematically manage, sort and combine data" (aSPECT3D, 2015).

2.1.3 Online viewer: the natural way to share $3 \mathrm{D}$ models to the public is the web, creating ad hoc on-line viewers and navigation systems. The main technology for $2 \mathrm{D} / 3 \mathrm{D}$ computer graphics on web is WebGL (Di Benedetto, et al., 2014). The available systems have big restriction on the size of $3 \mathrm{D}$ models that can be loaded; moreover, they usually are unidirectional

\footnotetext{
* Corresponding author
} 
tools created to show something without foreseeing any iteration with the system by the user.

3DHOP is one of the best example of " $a$ collection of tools and templates for the creation of multimedia interactive Web presentations"; it "allows easy visualization of $3 D$ models directly inside HTML pages and the streaming of multiresolution 3D meshes over HTTP" (3DHOP, 2015).

Nubes project developed by CNRS is a collection of four tools aimed mainly at specialist in $\mathrm{CH}$ conservation and valorisation. These tools permit to: i) have a database of 3D representation of $\mathrm{CH}$ at the architectonical scale, ii) display temporal changes, iii) make spatial references and semantic annotations of iconographic sources and iv) construct viewpoints that allow joining the previous tools. This project represents a platform specially designed for the digital analysis of architectural heritage (De Luca, at al., 2011).

\subsection{New proposal}

The lack of a complete integrated system, able to provide high modeling capabilities, dynamic data entry and wide data sharing at the same time, leads to the invention of the innovative system under Italian patent pending MI2014A002016 and its current implementation called BIM3DSG. It is created for the "advanced management, access to BIM (Build Information Model) data and $3 D$ visualization of models with high complexity, great accuracy (as it happens for example in the field of cultural heritage), heterogeneous and with different shapes (both line based and surface based), that cannot be currently managed in their entirety on the existing technology" (Fassi, et al., 2014).

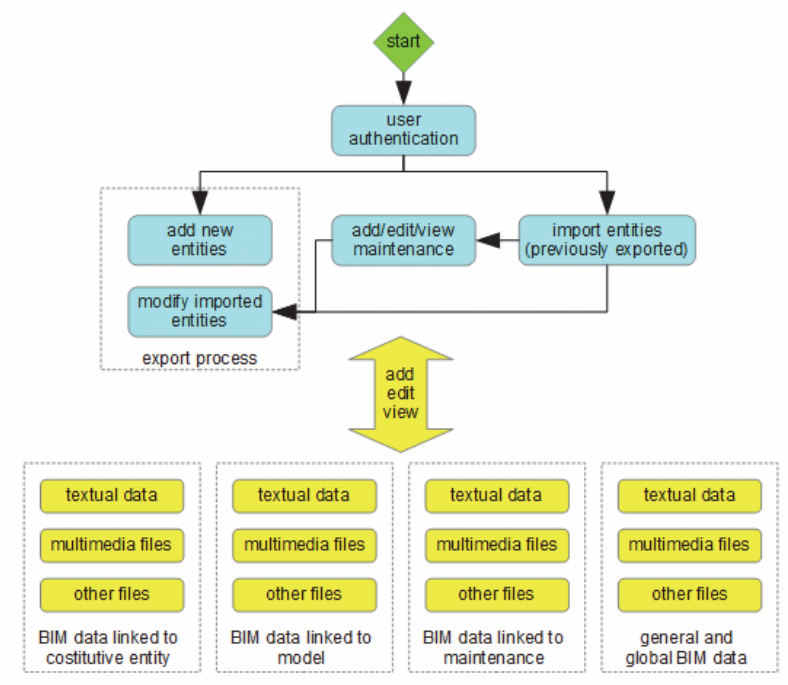

Figure 1. Block schema for the main features of BIM3DSG.

\section{BIM3DSG is divided in two parts (Fassi, at al., 2012):}

- $\quad$ the first one is necessary to add or modify $3 \mathrm{D}$ models into the system; it is thought to be mainly used from professional and 3D specialists. It can be a plug-in usable inside any modeling software or a standalone software. BIM3DSG supports every kind of previously modeled surfaces, both NURBS (Non Uniform Rational Basis-Splines) and Mesh, both of them with or without texture (Fassi, at al., 2010).

- the second part allows the use of the system through the web; it is thought for common users. It requires only a web browser and it is specifically designed for using it also on mobile device such as laptops, tablets, and smartphones, even characterized by low hardware resources.

Both sections let the user to access to the interesting parts, zones, sectors, areas, in addition to the whole model; the selection of desired objects can be done through many search functions, or can be obtained automatically through spatial and time relationships. Moreover, the operator can:

- view automatically computed BIM information of each object, such as area, volume, position,

- add/edit/view user BIM information and files (photo, video, documents, dwg, ...) associated with one or more objects and models,

- $\quad$ add/edit/view maintenance, restoration and building site activities and also all related information and files (photo, video, documents, dwg, ...),

- view past situation, and also view past and actual situation at the same time for comparison intent,

- view reports,

- define different access levels and user permissions for each user.

This paper focuses on the web browser visualization and on the information management system describing the system structure, the functionality and presents some different cases of study that space from architecture to museum application.

\section{BIM3DSG}

\subsection{Overview}

The base idea of the system is to identify three types of entity that can interact inside it:

- the "object", referencing the physical object,

- the "model" of the specific object, that is the 3D representation of the object at a determined time of its life, shaped from measured data in the survey phase,

- the "maintenance event", that is a real operation on one or more items that often modifies their structure.

Furthermore, the three-dimensional complex object is divided in a plurality of single elements (parts) that form it, each of them with its own 3D model.

Each element and each entity is stored individually, separated from the others, inside a central database (currently PostgreSQL); the database also contains the relationship between elements, entities and elements and entities. Moreover, all other data, both models and textures and information and additional files, are stored into the database.

Thanks to the central database, all functions can be used concurrently from different sites, both locally or remote, trough an internet connection. Diverse operators can work at the same time from different positions and all changes are shared in realtime; the system inserts a temporary write lock on objects that are under modifying, to avoid concurrency problems.

Due to the size of complex three-dimensional models (they can fill some Gigabytes), powerful caching mechanisms are implemented, for both parts, in order to ensure the userfriendliness and the appeal of the system, allowing almost instantaneous loading time, even on low speed network connection.

\subsection{Web Browser Models Visualization}

3.2.1 Introduction: one of the key aspects of BIM3DSG is the capability to use all features, except for the export process, inside a common web browser. 
This is very important for the diffusion and the fruition of the system by common users: web browser is familiar, it does not require any additional learning effort or software installation and it is OS independent. Moreover, it can be run on any device and it is perfect for mobility. All these characteristics help to persuade the consumers towards the fruition of the system during the daily work.

It is also fundamental for the dissemination: in fact, external users can access to the models and the related information without neither users nor organization bear any cost. To protect data and hide private information to the public, administrators can set read-only accounts and restrict user permissions (full access permission and separation, based on UNIX like schema, are supported by BIM3DSG).

BIM3DSG also provides, in addiction to keyboard shortcuts and mouse navigation, a complete touch interface; it makes it very simple to use on screen only device, such as tablets, smartphone, and it is perfect for interactive stations, e.g. inside a museum. The system offers complete controls to navigate, rotate and zoom inside the whole model; all controls are instantly usable, even when a huge scene is not yet completely loaded, to ensure no waiting time.

The user can select, through mouse or touch, one or more objects, merely clicking on them. After the selection, he can perform some actions on them, such as access to automatically computed BIM information, visualization or modification of custom BIM information and files associated with that object, addition maintenance events and visualization or modification information, images and files associated with each maintenance event.

To ensure the maximum interoperability and compatibility, the user interfaces is built using only HTML5, css, php, JavaScript, JQuery and Jquery Mobile (for some UI controls and icons), that are actually supported by most of browser and OS (JQuery Mobile, 2015), both for workstation and mobile device.

3.2.2 Loading models on web browser: the user can select the objects to view or load the entire model; the capability to load only the needed objects is very important because it lets to i) reduce loading time, ii) have better performance, iii) use high resolution and quality models even on low memory device, iv) not load useless object, v) create custom views, vi) facilitate the navigation through the model and vii) avoid errors.

The selection can be done by:

- using one or more filters on the parameters that identify the constitutive entities, for example the area, the zone, the sector, the type and the name of the single element,

- choosing a cross-section slice of the model, defining height and centre element,

- querying the system for elements inside a virtual sphere which radius and centre is chosen by the user,

- using one, more or ranges of codes, automatically associated by the system for each constitutive entities during the export process,

- using filters that involve maintenance, restoration and building site activities,

- choosing a particular historic period,

- reusing current user's last selection, that is automatically stored in the database for each account

During the selection procedure, it is possible to set the objects either in "write mode" or in "read-only mode". One element can be in "write mode" only for one operator at the same time, in order to avoid concurrency problems that can arise when multiple workers try to modify same data all at once.
The user can choose between seven different levels of detail (LoD), both for 3D models and textures. The lower LoD can be very convenient for loading an entire huge model on low memory device or when only a low bandwidth network is available and the models are not previously loaded on that device, so that they are not available into the system cache. A future improvement consists into the addition of an automatic mode, in which the system can dynamically switch the LoD of each element depending on its current screen size.

Regarding the texture visualization, the employer can, at any time, enable or disable it and choose texture LoD, regardless of the model's LoD.

During the import process, the system first builds the scene information: camera, lights and object's list; then it loads asynchronously each model, from the JSON and eventually texture files. For this process, BIM3DSG uses WebGL (WebGL, 2015) through SceneJS library (SceneJS, 2015), combining them with php and JQuery.

All needed files are locally cached into the browser persistent storage (Web Persistent Storage, 2015), consisting in a nonrelational database saved in a single disk file. For each file to be loaded, the system checks if an up-to-date copy is available into the persistent storage: in this case, BIM3DSG directly loads it from the local cache, saving time. Otherwise, before it can be loaded, it is necessary to read the file from the remote database and to store it into the persistent storage. Persistent storage is chosen because i) it is a standard browser capability, ii) it is not under the control of the browser cache manager, iii) it can have a custom size, iv) it is not removed when temporary files are cleared and v) it can be used to save data and information besides regular files.

3.2.3 Computation of the level of details for the web: the models at lower LoD are automatically computed during the export process, which currently requires the use of odelling management part.

The system uses a proprietary algorithm that decimates the mesh of each single element approximating the vertexes of the polygons on a 3D grid of increasing size (LoD1 $\rightarrow 1 \mathrm{~mm}$, LoD2 $\rightarrow 2 \mathrm{~mm}$, LoD3 $\rightarrow 4 \mathrm{~mm}$ and so on). As result, some vertices may overlap and thus they are replaced by a single approximated one, decreasing in this way their number and, consequently, the amount of polygons that will be recalculated at the end of the process.

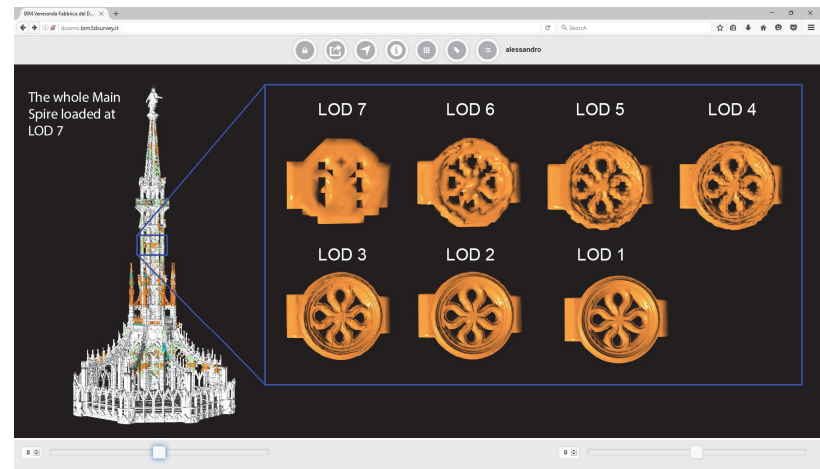

Figure 2. BIM3DSG web browser visualization of the same object at different LODs.

The LoD of the textures, instead, is obtained by resizing the image, using the pixel resize technique; the association between textures and models, the UV texture map, are preserved without 
the need of any further change, because it uses coordinates normalized between 0 and 1 .

The models are saved into JSON files, including also the UV coordinates, needed for applying the textures through the loading of the UV texture map (Mullen, 2009). Textures are saved as PNG or JPEG files. As told before, all files are stored into the database.

\subsection{Access to BIM data and files}

3.3.1 The information management system: the information management system is another key feature of BIM3DSG. In the BIM sector and even more in the $\mathrm{CH}$ field, there is the necessity to handle a lot of different kind of "not standard information". Each CH case of study is very different from others, the interpretation parameters are extremely variegated, concerning historical period, modifications through time, construction materials, decay, past and present restoration activities and all other singularities that make every heritage unique and not standardisable.

BIM3DSG is created on purpose to meet these multiple needs: it can be easily adapted to every user requirement with quick operations at the administration level. This is possible because it has not only a static base standard information system, but also a dynamic one.

The static system is used to display base standard information on the selected model: they are common to all case and are automatically computed during the export process and stored in the database. Most of them are related to the models and, for this reason, they cannot be manually modified, but are automatically updated if the model (or its property) of the object is changed. These information are divided into three groups: i) "Main info": area, zone, sector, type, name, version, current status (added/removed/modified during maintenance, unmodified, ...), creation and elimination date, ii) "Internal info", specifically related to the database: index number, model number, date of the last update and the user who makes that update and iii) "Model info": area of external surface, volume, centroid coordinates and bounding box dimension.

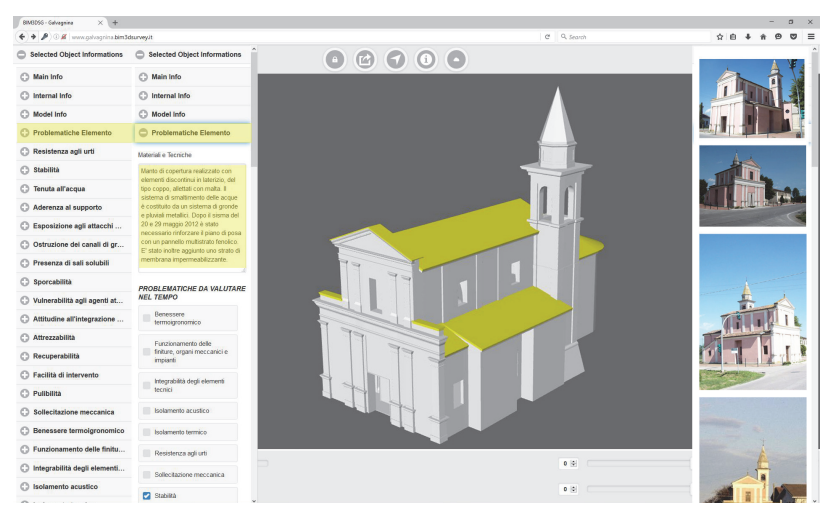

Figure 3. BIM3DSG dynamic information for the web. Groups and fields are created dynamically, reading them from the database, so that their changes are retrieved almost in real time.

Regarding the dynamic system, BIM3DSG offers the capability to define quickly new custom groups (logical tables) of information, adding them to the database; for each custom group, it is possible to define personalized fields of information, adding them to the database specifying a label and the desired data type. Each project owns a different database instance that contains its own information groups and fields, so that they can be easily added or modified, adapting them to every specific needed content.

BIM3DSG supports any type of data entry: each personalized fields can contain text, integer, float, boolean (checkbox) and date-time. There is no limit both for the number of groups and for the number of fields inside each group. Thanks to these characteristics, the system is able to manage easily any possible requirement.

From the user point of view, each costumed group is shown in the same way as the basic ones (grouping them with expanders inside web, with dockable floating windows or tabs for Rhino). To facilitate readable and pleasant visual formatting, there is also the possibility to add titles text, separators and links, an interesting feature that lets to link a different custom group and thus build custom info-trees.

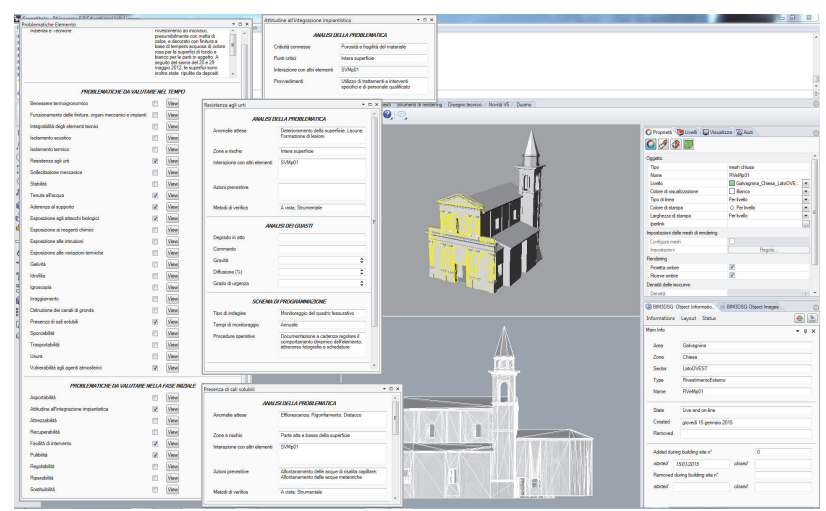

Figure 4. BIM3DSG dynamic information at work inside the modelling management part.

Each information can be linked both to an object or to a model or to a maintenance event, according to the requirements and without any restriction on the kind that can be added to the system. Beyond the typical BIM data, users can request to insert any other type of record: for example historic, production, maintenance, planning and facility management.

Every group of custom information can be either related to a single entity or shared with many others, keeping their own separate identities than the other groups. This helps the operation of "information assignment" when the user needs to attribute the same information to a huge number of entities.

Through this dynamic system, the operator can add and visualize any type of custom information and the system can be quickly and easily upgraded to adapt to new needs and requirements.

3.3.2 Multimedia and additional file support: in addition to the information system, BIM3DSG offers the possibility to insert external files, e.g. images, videos, documents, CAD files. Conceptually, file support can be used in the same way of the information system: after selecting one object, the user can view and modify previous inserted files or add one or more new files. As for the information system, each file can be linked to an object, to a model or to a maintenance event and can be individually shared with other entities or group of entities too. The system employs two different panels for files. The first one includes the multimedia data, showing a low-resolution preview for images or the first compressed frame for video: when the user clicks on it, he can view the image at full resolution or start the video playback at the original quality. For each multimedia element, the system supports the input of an optional description, modifiable by the operator. 
The second panel is for non-multimedia files, displaying a "fileexplorer-like" interface. When the user clicks on a file icon, it is opened outside the browser, using the default system program for that extension.

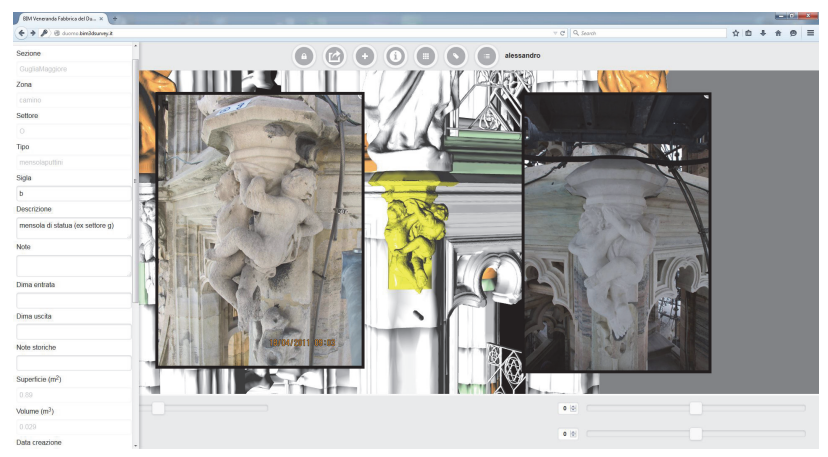

Figure 5. BIM3DSG multimedia gallery at work.

BIM3DSG leaves the caching process, for preview, images and files, to the browser (standard caching technique), even if it is under evaluation the benefit to manage it in the same way of JSON files, thus adding them to the persistent storage.

\subsection{Maintenance activities}

BIM3DSG has at disposal a full complex system to handle maintenance, restoration and building site activities; they are fully supported also through the web interface.

In order to keep at the same time the history of the objects and their geometrical transformations, the system creates new entities referred to the new situation, while keeping the previous ones.

BIM3DSG supports any type of relationship between previous and new objects: one to one, one to many, many to one, many to many. When the user adds a new maintenance event, the system automatically creates the structure for: i) the activity, ii) the new objects and iii) the information and additional files about the operation.

Some maintenance events change form and geometry of the elements, instead others have not effect on the structure; the system is able to take care of both problems. In the first case, the operator must insert the new models characterized by new geometries and physical form. Otherwise, the system automatically uses the old ones, without duplication; to comply with this requirement, BIM3DSG supports the capability to use one single model for different objects.

\section{CASE STUDY}

\subsection{Overview}

The BIM3DSG system has been tested in different case studies to visualize and manage many different types of models, from architectural to archaeological ones. In the following paragraphs are described four different examples concerning two architectonical buildings, one archaeological site and an object belonging to statue field.

They are different from many points of view: the scale of the objects, the final resolution, the survey method in which they are recorded and the presence or absence of textures. The goal is to test the versatility of the system and its ability to self-adapt to the different needs characterizing the world of $\mathrm{CH}$.

\subsection{Milan Cathedral: large and complex architectonical building}

The architectonical case is the "Yards of Milan Cathedral" (Achille, at al., 2012). This is an important example of a very complex and large structure, composed by a huge number of single parts.

The typical problems encountered are:

- the large amount of elements to be modelled (4.825 for the main spire, 3.358 for the north altar, 2.883 for the south altar, 379 for the $18^{\text {th }}$ spire and 4.194 for the dome cladding),

- the different type of objects: simple ones (such as pillars, arches, ribs and buttress), that are the outcome of extrusion of surfaces along lines, and, on the other side, complex artistic pieces, that comes from retopology operations,

- the real time collaboration among different operators in the survey phase, in the modelling phase and in the usage of the final model,

- the use of the BIM system directly on the yard,

- the maintenance operations that modify radically the form and the geometry of the pieces.

Due to the large amount of modelled objects, for each yard it is mandatory to have the possibility to choose which part of the model the user wants to display, in an interactive manner. This feature is very important because:

- portable devices used in the yard have not high computing power,

- load the whole model every time involves waste of time,

- $\quad$ load a large number of models can lead to additional costs, due to data connection,

- it is easier to identify the inner blocks of the building.

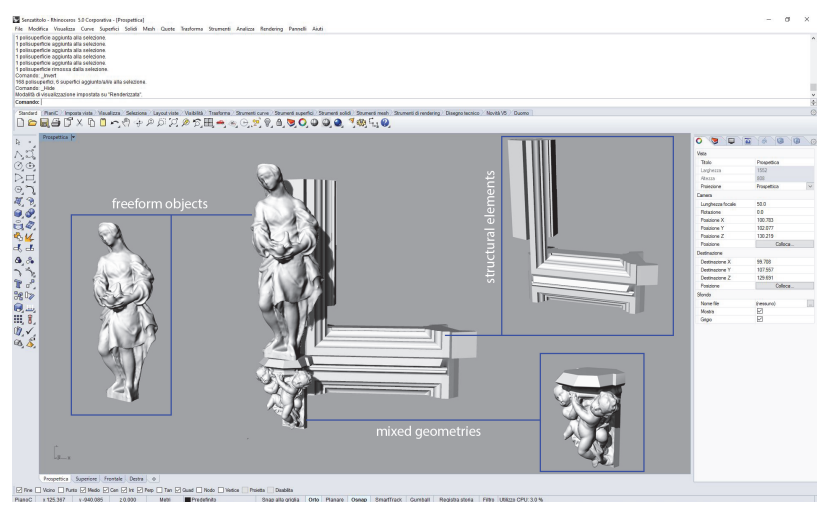

Figure 6 . Three types of geometry: 1. Free form coming from re-topology operation, 2. Extrusion of surface along line, 3. Mixed geometries.

Due to this requirement it was implemented the previous described feature that lets to load the elements by yard (area), by zone of the yard, sector of the yard, type of the object and the name of the object related to the previous fields. (Example: GugliaMaggiore(area) - Camino(zone) _ M(sector) Pilastro(type)_ 16(name). This structure of layers prevents any ambiguity of selection. Moreover, in order to clarify which blocks are involved in a specific maintenance operation, it is possible to load only the models closer to the one interested by the intervention. The selection can be made through a cross section, a sphere around, the level of scaffolds, ranges of unique codes associated to the models. 
In this case, the key point of the system is the possibility to manage all the interventions regarding maintenance and restoration activities. The management of the interventions can be done through the web interface of BIM3DSG, where the user can select one or more imported elements of the whole model and create the intervention. Mainly there are three types of interventions: cleaning of marble surfaces, entire and partial replacing of marble objects. These, due to yard's reasons, can involve one or multiple parts at the same time. The ability to manage different kinds of relationship between the elements, designed inside the BIM3DSG system, covers precisely this need.

Both for workers and for the director of the yard, it is important to have an immediate perception of the progress of restoration on a specific date. The coding adopted to visualize quickly the interventions operates through colours: blue when an intervention is created on an object, green when it survived to the intervention, red when a new one is added.

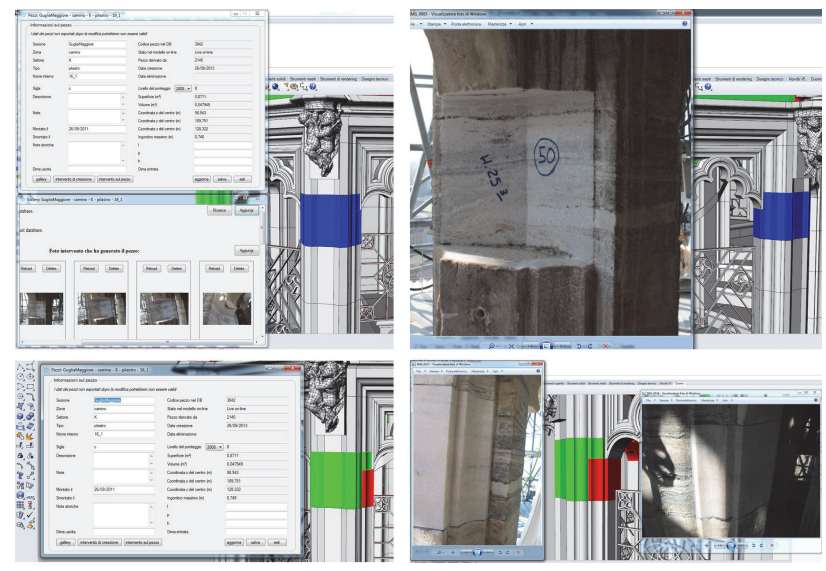

Figure 7. Colour code for interventions.

Some other information are automatically "calculated", together with the colours, for example, the date in which the operation starts, a code that uniquely identifies that piece of model, the area, the volume, the weight, the centre of gravity and the bounding box dimension. These are useful when it is necessary to disassemble some marble blocks, so it is possible to prepare the necessary machineries to complete the works.
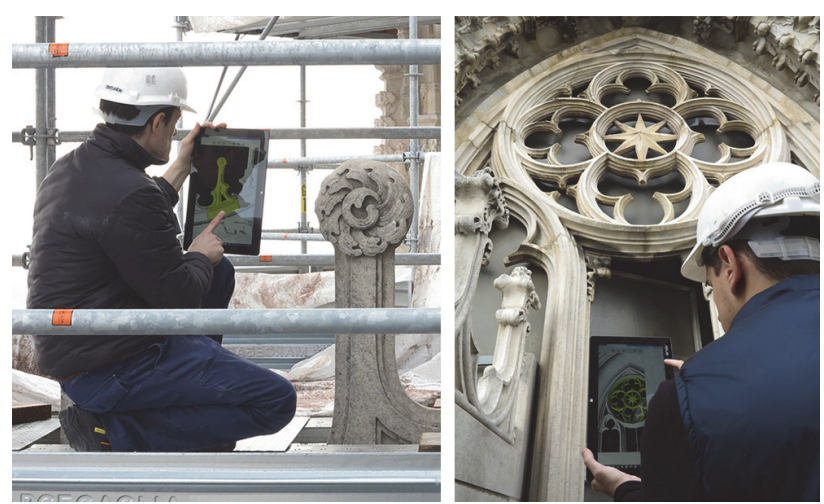

Figure 8. Use of BIM3DSG in the yard.

Moreover, the system lets users to add data and notes both to the blocks and to the interventions created, in particular regarding the historical information, the date of conclusion of restoration process, the description on how the job was done, the used products and the problems encountered. It is also possible to link images or video, before, during and after the restoration, and any other files. The information on the dates allow loading models at different time thresholds, giving a further opportunity to follow the progress of work.

As a positive result, the workers of Veneranda Fabbrica del Duomo use the system in the yard for daily operation of restoration or maintenance, sharing the information in real time with the technical office, which has the task of updating the models according to the indications coming from the BIM3DSG's web site.

\subsection{Galvagnina}

The second architectonical case regards a church built in the XVI century located in Pegonaga (MN) that was seriously damaged during the earthquake of 20th and 29th May 2012. According to the Italian regulations (D.P.R. 207/2010) it was necessary to produce the Planned Preventive Maintenance Program (PPMP). The document includes the verifications to be carried out and all information for maintenance during the following years.

In this case, BIM3DSG was tested in order to support this document confident that WEBBIM processes can effectively contribute to the management of historical and archaeological heritages; for this purpose, the model, built in REVIT, was uploaded on BIM3DSG.

In a following phase ad-hoc tables were linked to different parts (walls, floors, roofs, windows, etc...), the information contained regard problems, causes of the decay, state of conservation and actions to be performed.

The positive result is represented by the possibility to include both model and information in a unique database outside commercial software; this allows operators involved in the PPMP to take part in the decision stage and follow the development of works.

\subsection{Umm El Dabadib (Rossi, 2013)}

The second case deals with the possibility to visualize highresolution and textured mesh of very large objects belonging to archaeological heritage.

In archaeological field the use of a BIM system, like BIM3DSG, is very useful because is strong effective in presenting $3 \mathrm{D}$ surveys and their elaborations. The possibility to view and study data on line makes the knowledge of archaeological sites accessible to everyone; moreover, the 3D domain is more effective than bi-dimensional drawings that are quite a standard in this field.

BIM3DSG offers an on-line informative system, in which the entire 3D survey of Umm El Dabadib can be uploaded, studied and used by other researchers. In addition, this system is able to record not only the existing remains, but also their evolution over the years, keeping tracks of the changes that might occur due to natural events or artificial interventions (decay, damage, conservation works, etc.).

The case study presented is about a Roman fortified settlement of 300 A.D. in the Western Desert of Egypt; the site is very hard to reach and it was unknown until some years ago. It is located $800 \mathrm{~km}$ south far from Il Cairo and $200 \mathrm{~km}$ west from Luxor, the archaeological site covers an area of one ha. The models obtained and discussed here are the result of two campaigns in which the entire settlement was surveyed only with photogrammetry (Fassi, at al., 2015).

Unfortunately, year by year the remains reduce their dimension because of collapses, human destructions, climate events and erosion of the desert. In such a case, it is important to have at 
disposal an accurate 3D model in order to study it, make some prevision or hypothesis on its restoration without the need to go there. Only few months per year are optimal to stay on site, as in spring the strong winds makes work very hard and in summer the desert is too hot. Moreover, in the last years some archaeological missions were forbidden due to political reasons. In this case, relevant information concerning the history of the settlement, his evolution through the years, historical images, videos exploration for study purposes and orthophoto (extracted from the model) are inserted and linked to the objects. A standard metadata information group is currently under development.

The models that are now possible to view and share simultaneously or one by one are 18 . Every piece of model is about a square of side $25 \mathrm{~m}$ and they are placed in a grid of 4 columns by four rows. One model is completely dedicated to the central castle of the settlement and one is a rigid ring around the square of $100 \mathrm{~m}$ per $100 \mathrm{~m}$. Single models have an average of 3 million triangles and are textured with high resolution images reaching the atlas of 8192 x 8192 pixels. The segmentation of the model makes possible to assign different information to each "block", so a set images representing very interesting artefacts found in a specific place can be placed in the right position. Moreover, detailed plans and section of particular buildings will be linked with the relative one, this feature permits to "georefernce" data.

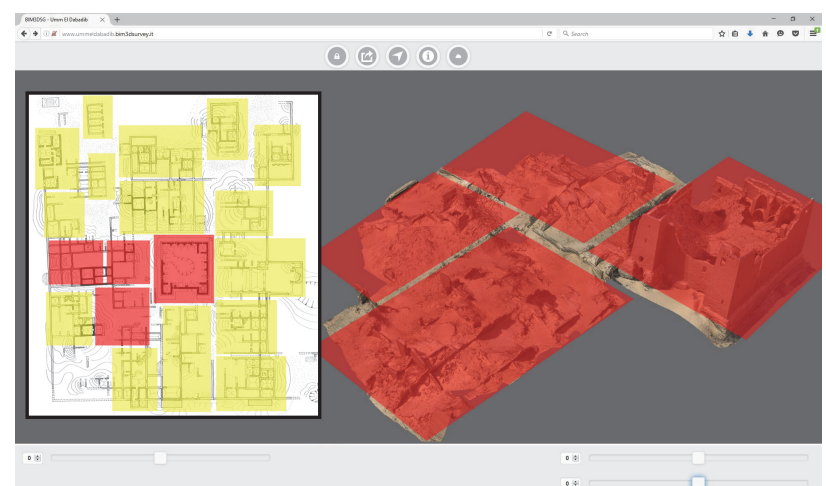

Figure 9. The image shows simultaneously 4 of the 18 buildings in BIM3DSG system.

The use of BIM3DSG in this case lets to create a knowledge platform in which the results of the work can be uploaded and examined by others experts. The use of this method can benefit other colleagues with similar problems and other sites under threat.

In these particular case of very large sites, the main issues, that are partly resolved by BIM3DSG, are:

- the huge quantity of data to display,

- the dimension of the site, that creates navigation problems,

- the resolution of the texture,

- the segmentation of the models,

- the necessity of a standard metadata information.

\subsection{Statue and decorations}

BIM3DSG system are quite ready to be used as catalogue of artefacts, such as statues decoration and little objects museum items. It is important to record the goods not only in order to have an index of artworks (typical of virtual museum), but also to have data regarding conservation status of the objects (Incerti, at al., 2014). Moreover, if the artworks are recorded both before and after a restoration is possible to have an historic $\log$ of activities.

The case studies that already works on BIM3DSG are several, here is presented an emblematic one because of its dimensions (quite small) but very detailed (the harnesses of the horse or the armature of the cavalier). The bronze equine statue that belongs to a private collection is about $57 \mathrm{~cm}$ tall, $54 \mathrm{~cm}$ long and 30 $\mathrm{cm}$ wide. It was surveyed with photogrammetric approach using a Canon 5D Mark III, 35mm lens in order to have a very detailed model at 1:1 rendering scale. The final result is a very high-density mesh of about 5 million of triangles; the texture is obtained reprojecting high resolution images on the model (the atlas is $8192 \times 8192$ pixels).

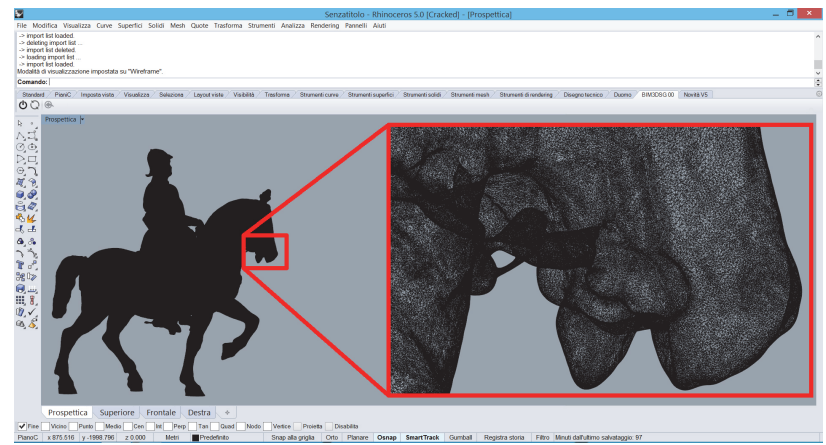

Figure 10. A detail of the mesh.

In these cases of study is necessary to have at disposal a system able to:

- visualize very high resolution $3 \mathrm{D}$ models and textures,

- $\quad$ provide a robust database of information, able to store big quantity of data,

- grant standard metadata and paradata schemas,

- involve users in active fruition of artefacts through a clear interface.

The term metadata refers to the information of the physical object (such as surface, volume, dimensions, material, etc.), paradata instead are the features that describe its model (instruments used to extract the metadata, date of survey, operator, modelling info, etc.).

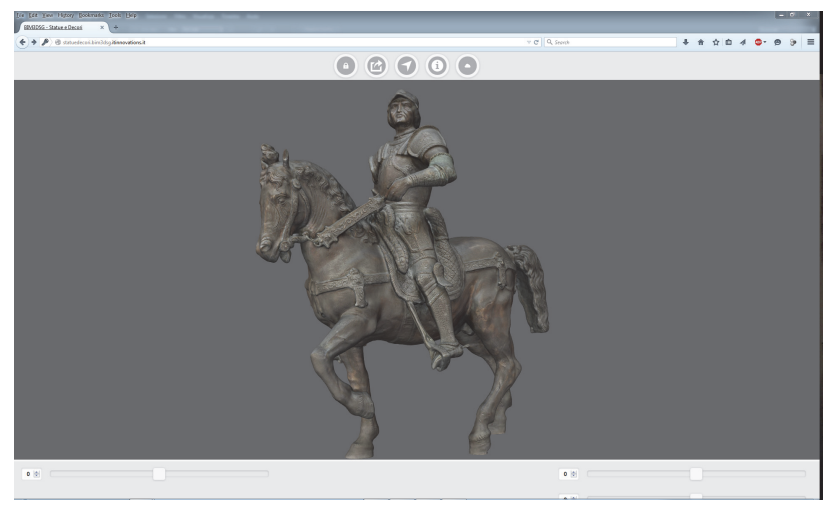

Figure 11. The statue inside BIM3DSG, the view is centre on the object and the sliders below permit the standard actions of zoom and rotation. The user friendly icons in the upper part let to see info and images.

In this case, the key aspect is the capability of the system to distinguish between physical object and model and the ability to relate different groups of information between them. 


\section{CONCLUSION}

Many experiences in the field of $\mathrm{CH}$ have grown a strong need of dedicated information systems created ad hoc for the managing of survey data, 3D representation, restoration operation and different kind of information related to the objects, to the models and to the maintenance activities. These type of systems can be used both to present the final work of virtual reconstruction and as support for any activities on the heritages and for the facility management of buildings.

Generally, the main requirements are the possibility to manage very complex, extensive objects and high-resolution models, to cooperate with different stakeholder in real time and to make available the results of the work to the public. BIM3DSG, here presented, gives some interesting positive answers and solutions. Different cases of studies testify as the system can be easily used not only in architectonical field and extensive archaeological cases, but also for little objects and museum applications.

The Its key aspect of the system is the possibility to interact with the model using the web. It is not only mere visualization, but also the possibility to "read" and "write" information. In this sense, the system allows wide collaborative activities and real time data updating. Some features, as the possibility to manage multiple complex relation between objects and to handle "Objects" and "Models" in different way, makes the system highly flexible, able to adapt itself to any possible situation.

In order to improve it and his spread, some further developments are planned both for the modeling management part and for the web browser visualization.

The main idea is to generalize the use of the system, developing: i) similar plug-ins inside other modeling software, ii) a standalone software that gives users the ability to export OBJ files into the system without depending on a modeling software and iii) a web service that gives users the ability to export OBJ files into the system directly through the web interface.

On the web side, additional features are currently under development: i) an automatic LoD mode, in which the LoD of the models for each object are automatically chosen and dynamically changed in relation to the zoom, ii) a refined mesh decimation algorithm, iii) the possibility to insert region of interest as part of an object, iv) the capability to add information, images and files to each region and v) some measuring tools inside the web browser.

\section{ACKNOWLEDGEMENTS}

Big thanks to Stefano Parri (New Tread s.r.l, Italy), a friend who works with us to this project for many years and gives us many great ideas on how realize and improve this system.

\section{REFERENCES}

Fassi, F., Achille, C., Mandelli, A., Rechichi, F. \& Parri, S., 2015. A new idea of BIM system for visualization, web sharing and using huge complex 3D models for facility management. In: 3DArch, Avila.

Revit, Autodesk, online at http://www.autodesk.it

aSPECT3D, Arctron, online at http://aspect3d.arctron.de
Di Benedetto, M., Ponchio, F., Malomo, L., Callieri, M., Dellepiane, M., Cignoni, P. \& Scopigno, R., 2014. Web and Mobile Visualization for Cultural Heritage. In: Lecture Notes on Computer Science LNCS 8355, Springer, pp. 18-35.

\section{DHOP, online at http://veg.isti.cnr.it/3dhop/}

De Luca, L., Busayarat, C., Stefani, C., Véron, P. \& Florenzano, M., 2102. A semantic-based platform for the digital analysis of architectural heritage. In: Computers \& Graphics, Vol. 35(2), pp. 227-241.

Fassi, F., Rechichi, F. \& Parri, S., 2014. Metodo e sistema per la gestione e la visualizzazione di modelli di oggetti tridimensionali complessi. In: Italian patent pending MI2014A002016.

Fassi, F. \& Parri, S., 2012. Complex architecture in 3D: from survey to web. In: International Journal of Heritage in the digital era, Vol. 1, pp. 379-398.

Fassi, F., Achille, C., Fregonese, L., Monti, C., 2010. Multiple data source for survey and modeling of very complex architecture. In: International Archives of Photogrammetry, Remote Sensing and Spatial Information Sciences, Newcastle upon Tyne, Commission V Symposium, Vol. XXXVIII, Part 5.

JQuery Mobile, online at https://jquerymobile.com

WebGL, online at https://get.webgl.org

SceneJS, online at http://scenejs.org

Web Persistent Storage, online at http://www.w3.org/TR/webstorage

Mullen, T., 2009. Mastering Blender. Wiley Publishing, Inc, Indianapolis, (1).

Rechichi, F., ITinnovations Library, online at http://www.itinnovations.it, 2014

Xceed WPF Toolkit and AvalonDock, online at http://wpftoolkit.codeplex.com, 2007

Achille, C., Fassi, F. \& Fregonese, L., 2012. 4 Years History: from 2D to BIM for $\mathrm{CH}$. The main spire on Milan Cathedral. In: G. Guidi, A. C. Addison. Proceeding of VSMM 2012, Virtual Systems in the Information Society, Milan, pp. 377-382.

Rossi, C., 2013. Controlling the borders of the empire: the distribution of Late-Roman 'forts' in the Kharga oasis. In: Bagnall R., Davoli P, and Hope C. (eds.) The Oasis Papers 6, Proceedings of the Sixth Conference of the Dakhla Oasis Project, Dakhla Oasis Project Monograph 15, Oxford: Oxbow.

Fassi, F., Rossi, C., Mandelli, A., 2015. Emergency survey of remote and endangered archaeological sites. In: Int. Arch. Photogramm. Remote Sens. Spatial Inf. Sci., XL-5/W4, pp. 8591

Incerti, M., Iurilli, S., 2014. From survey data to virtual environment. Two case studies. In M. SCIentific RESearch and Information Technology Ricerca Scientifica e Tecnologie dell'Informazione, Vol. 4(2), pp. 87-108 